\title{
Percutaneous thrombectomy using a novel single session device for acute iliofemoral deep vein thrombosis
}

\author{
Gerard O’Sullivan(1) \\ University Hospital Galway, Ireland
}

\begin{abstract}
Summary: A 74-year-old woman presented with acute symptomatic left thigh and calf swelling; imaging demonstrated evidence of occlusive thrombosis from the upper left common iliac vein to the mid-thigh. Single session zero-thrombolysis venous thrombectomy was performed using the ReVene ${ }^{T M}$ Thrombectomy Catheter.
\end{abstract}

Keywords: Iliofemoral deep vein thrombosis, single session mechanical thrombectomy, quality of life

\section{Introduction}

The treatment of venous disease is gaining increasing importance. While the arterial endovascular management of peripheral arterial disease has progressed rapidly in the past two decades, venous disease is at a relatively early stage with the exception of a few dedicated centres [1]. Venous thromboembolism (VTE) is the third most common cardiovascular cause of death after heart attack and stroke [2] Deep Vein Thrombosis (DVT) can be associated with acute complications such as pulmonary embolism; and longer-term complications such as post-thrombotic syndrome (PTS) [3]. Traditionally, primary treatment for DVT has focused on anticoagulation, rather than thrombus removal, despite the fact that anticoagulation therapy does not remove existing thrombus, but rather prevents further propagation of the thrombus [4].

While anticoagulation therapy remains the standard of care for patients with deep vein thrombosis, Pharmacochemical catheter directed thrombolysis (PCDT) has been shown to reduce acute leg pain and improve quality of life in patients with acute iliofemoral DVT [5].

\section{Case report}

A 74-year-old woman presented with a severely swollen left thigh and calf, she was symptomatic for two weeks. The patent was enrolled in the Venous Thrombus Extraction
(VETEX) Clinical Study: A Preliminary Investigation. This study is an open label, prospective non-randomised, multi-centre first-in-human evaluation of the Vetex ReVene ${ }^{\mathrm{TM}}$ Thrombectomy Catheter for treatment of acute iliofemoral DVT. The study objective is to evaluate the safety and performance of the device.

The primary performance endpoint of the study was procedural success which is defined as successful preparation and use of the device to achieve Society of Interventional Radiology (SIR) Grade II Lysis (50-95\% thrombus removal) in the target vessel, with freedom from procedural related adverse events. The secondary endpoints include calf circumference, patient quality of life and venous disease severity assessments, i.e. Villalta Score, Venous Clinical Severity Score (VCSS) and the VEINES-QOL/Sym score.

The study was approved by the relevant competent authorities and ethical committees and was performed in accordance with the guidelines of good clinical practice (GCP) and applicable regulations, including the Declaration of Helsinki. The patient provided written informed consent prior to enrolling into the study.

The patient had a significant history of gallstones and ascending cholangitis prior to presentation of DVT symptoms. A computed tomography venogram (CTV) indicated an occlusive thrombus extending from the left common iliac vein (CIV) all the way to the lower femoral vein (FV) (Figure 1).

\section{Device description}

The ReVene ${ }^{\mathrm{TM}}$ Thrombectomy Catheter is a 10 French thrombectomy catheter with patented cage technology designed to remove a significant volume of thrombus from iliofemoral veins without the need for thrombolytics. It is intuitive to set-up and operate, and no capital equipment is required (Figure 2).

\section{Procedural details}

The popliteal vein was accessed using ultrasound guidance; initial venography through a 10 French sheath 


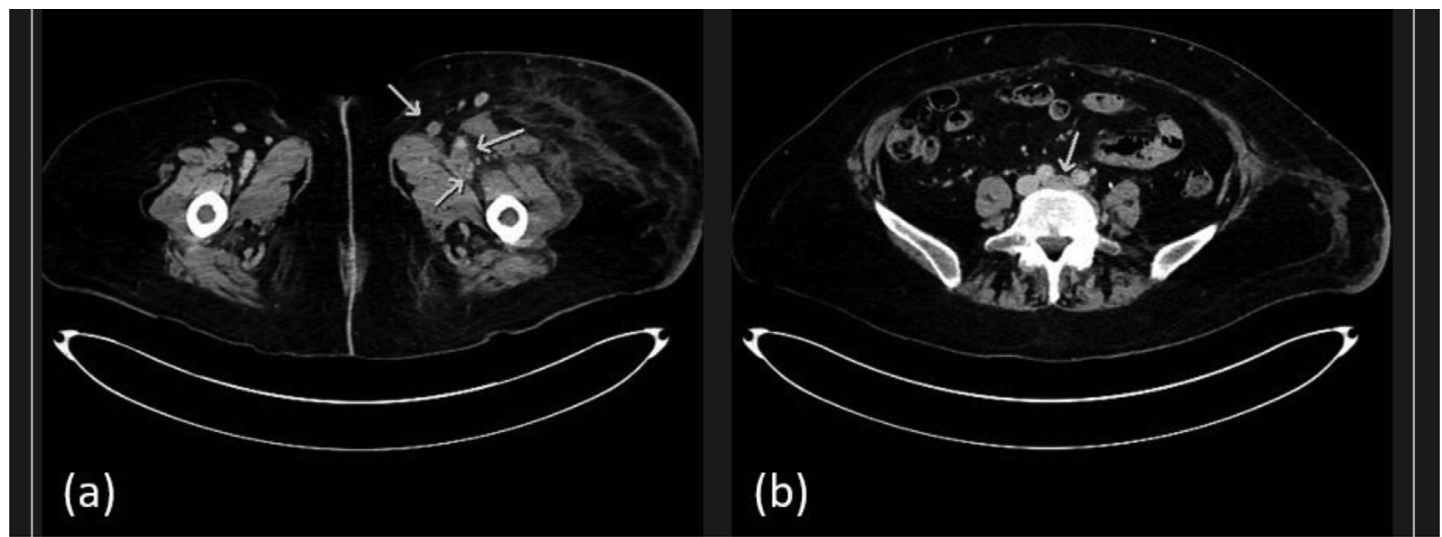

Figure 1. (a) CTV showing acute left femoral vein (FV), profunda vein (PFV) and great saphenous vein (GSV) thrombus and (b) acute left common illiac vein (CIV) thrombus.

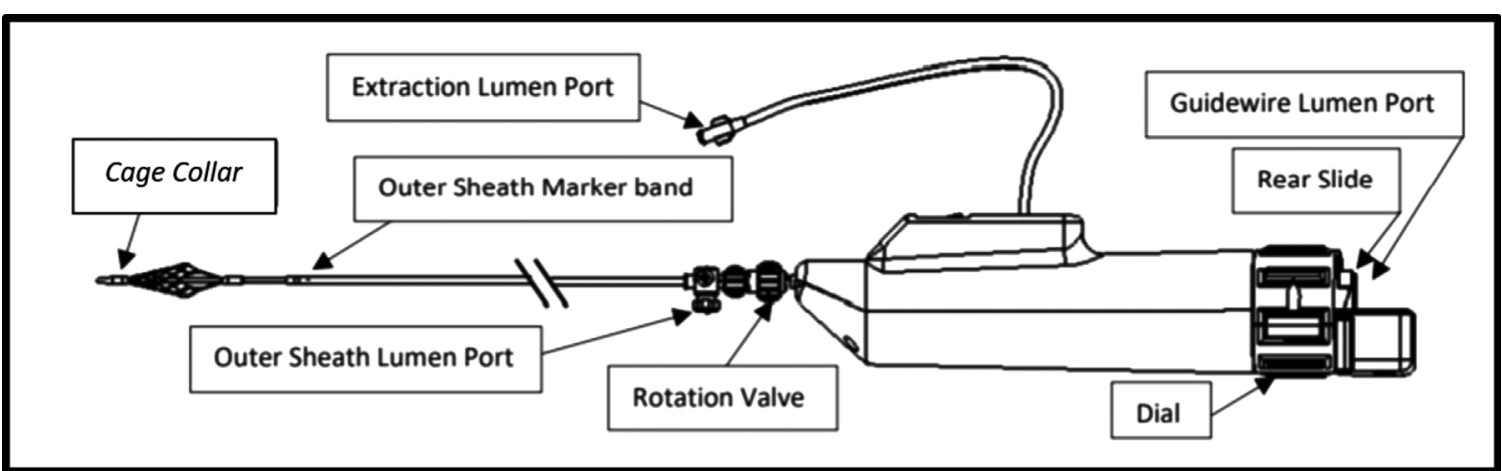

Figure 2. Schematic of ReVene ${ }^{\mathrm{TM}}$ thrombectomy catheter.

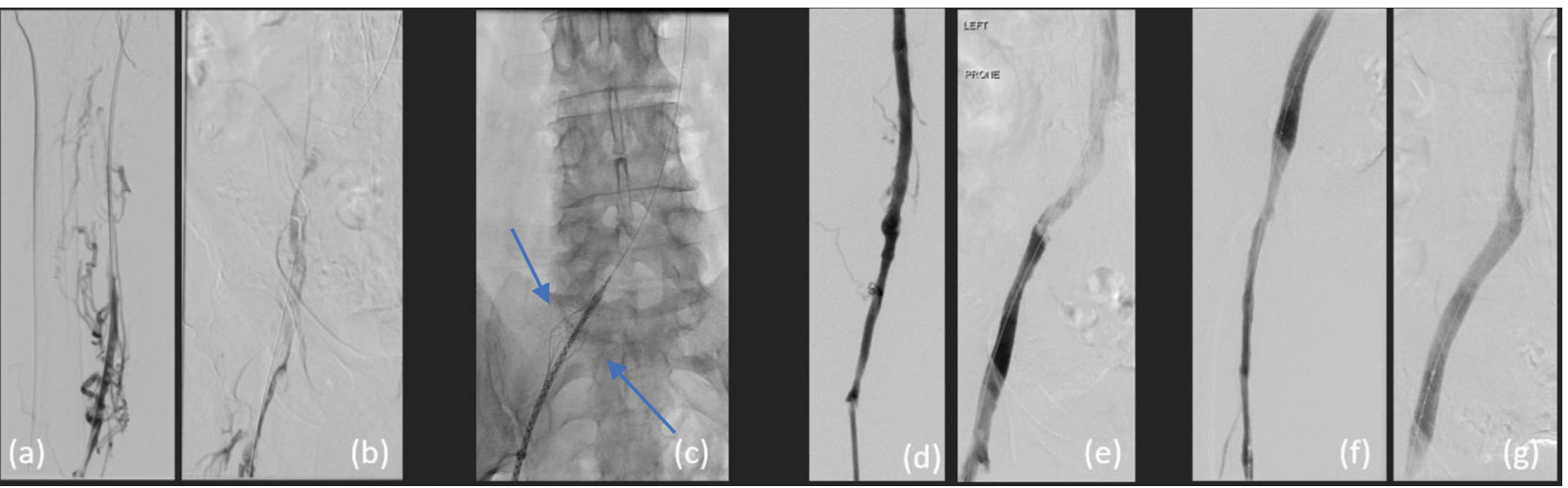

Figure 3. (a \& b) Pre-thrombectomy (c) Patent cage technology expanded ( $\&$ \& e) In line flow restored pre-stenting (f \& g) post stenting.

demonstrated a degree of chronicity to the thrombus in the lower femoral vein at the level of the adductor canal with collaterals passing to a relatively well developed profunda femoris vein. All deep veins from the left adductor canal to the upper common iliac vein were thrombosed (Figure 3). Five thousand (5000) units of heparin was given systemically to maintain an activated clotting time (ACT) level of $>250$ seconds. A retrievable inferior vena cava (IVC) filter was inserted into the IVC via popliteal access (in line with the hospital protocol for using any new thrombectomy device). The catheter was inserted over a guidewire and advanced past the treatment site. The device was unsheathed, and the cage was opened by rotating the dial on the handle (Figure 3). The extraction system was activated, and the device was slowly withdrawn in a retrograde direction from cranial to caudal through the thrombus, with the cage self-adapting to the vessel size to maintain wall-towall contact throughout. Once the first pass was completed the cage was collapsed after extraction by rotating the dial, the outer sheath advanced forward and the device removed. A total of two passes of the device were completed of the thrombosed segments; , the entire thrombectomy procedure was completed in 20 minutes which included including IVUS and venograms. Society of Interventional Radiology (SIR) grade III thrombus removal was achieved, indicating thrombus removal greater than 
Table I. Calf circumference, VEINS quality of life (QOL) score and venous disease scorings at baseline and follow-up

\begin{tabular}{lccc}
\hline & Calf circumference $(\mathrm{cm})$ & VEINS QoL/SYM & Villalta score \\
\hline Baseline & 50 & 74 & 10 \\
24hr follow up & 41 & - & - \\
1-month follow up & 46 & 104 & 1 \\
6-month follow up & - & 102 & 0 \\
\hline
\end{tabular}

95\%. No thrombolytic agent was used. Post thrombectomy venography revealed in-line flow had been restored as far as the left iliac system (Figure 3) with a persistent, chronic, high-grade stenosis in the left common iliac vein (L CIV), the probable cause of the DVT. Pre-stent venoplasty of the high-grade stenosis was performed to $14 \mathrm{~mm}$ diameter at $20 \mathrm{~atm}$. A Venovo ${ }^{\mathrm{TM}}$ stent $(14 \mathrm{~mm} \times 120 \mathrm{~mm})$ was deployed, and the stent was post = dilated using a $14 \mathrm{~mm}$ Atlas $^{\mathrm{TM}}$ balloon at $20 \mathrm{~atm}$. On completion, venography looked excellent and these findings were confirmed using IVUS. At 6-month follow up, the patient had no significant leg pain and showed equal sized calves and thighs, in addition to significant improvements in VEINS-QoL and VCSS scores and showed a Villalta score of 0 (Table I). Day 1 ultrasound demonstrated widely patent left lower extremity veins; and the patient was discharged on full anticoagulation; initially in the form of low molecular weight heparin followed by a NOAC (Eliquis $5 \mathrm{mg}$ bd) up to 6 months. The filter was removed at 4 months - delayed due to COVID and again widely patent veins were seen. Finally at 6 months ultrasound of the left lower extremity revealed widely patent veins from below the popliteal to the IVC.

\section{Discussion}

The treatment for iliofemoral DVT ranges from medical management to use of adjunctive endovascular therapies for thrombus removal [6]. In this case, the conversation with the patient focused on the mechanical removal of thrombus without the requirement for thrombolytics. The use of thrombolysis increases the bleeding risk for patients, and it uses significant hospital resources in terms of bed usage and cost. In this current era of medicine post Covid-19 outbreak, shortening hospital stays and freeing up intensive care units (ICU) beds has become increasingly important. Single session thrombectomy treatment with this catheter, without the requirement for thrombolytics, was a safe and effective option for this patient with iliofemoral DVT.

Acute iliofemoral DVT is a major cause for morbidity, as approximately half the patients managed conservatively with anticoagulation therapy and compression stockings develop post thrombotic syndrome (PTS) [7-10]. The residual thrombus load correlates with the probability of a future PTS $[11,12]$ and the degree of the PTS correlates with the impairment in quality of life $[13,14]$.

Compared to conservative therapy, catheter directed thrombolysis (CDT) is associated with greater venous patency and reduced risk of PTS at two years [15] but is associated with a bleeding risk [16]. International consensus guidelines recommend CDT with provisional stent placement of the underlying venous outflow obstruction for selected patients with acute iliofemoral DVT [17, 18]. This patient was stented with a Venono ${ }^{\circledR}$ stent which following successful single-session thrombectomy.

Over the years a number of different assessments have been developed to assess quality of life and assist in the diagnosis of PTS $[4,19]$. The Villalta Score was developed specifically to diagnose and categorise the severity of PTS and has been used in clinical studies for this purpose [19]. The American Venous Forum developed the Venous Clinical Severity Score (VCSS) scoring system, however it is not as commonly used for PTS. VCSS assesses the severity of pain or discomfort, clinical symptoms and the frequency of compression therapy use [19]. The VEINESQoL/SYM is a patient-reported questionnaire and it has been cross-validated with clinical examination. This patient showed improvements across all 3 evaluation methods, with VCSS score decreasing, the Villalta Score of 0 at 6 month follow up indicating no Post Thrombotic Syndrome (PTS) and the VEINES-QoL/SYM score increasing at both 1-month and 6-months post-treatment compared with baseline. These evaluation methods are also supported by a reduction in calf circumference from $50 \mathrm{~cm}$ at baseline to $46 \mathrm{~cm}$ at 1 month follow up. The results from this case study as part of the VETEX study correlate with the findings of other studies, whereby removal of thrombus using mechanical and/or pharmacomechanical methods reduced leg symptoms [20].

\section{Conclusions}

This case report, which was performed as part of the VETEX study, provides an initial indication that the Vetex ReVene $^{\mathrm{TM}}$ Thrombectomy Catheter is a safe and an effective option for removal of thrombus in acute iliofemoral DVT patients. The treatment provides rapid restoration of blood flow in a single session with the absence of device related complications out to 6-months post-treatment. The case report also demonstrated a marked improvement in both quality of life and a reduction in disease state scoring.

\section{References}

1. Kapadia S, Chew D, Cura F, L'Allier P, Roffi M, Tuzcu EM. Textbook of Interventional Cardiology, A Global Perspective. First edition. Jaypee Brothers Medical Pub; 2017. 
2. Kevane B, Day M, Bannon N, et al. Venous thromboembolism incidence in the Ireland east hospital group: a retrospective 22-month observational study. BMJ Open. 2019;9(6):e030059.

3. Bozkurt A, Kırbaş I, Kösehan D, Demirçelik B, Nazlı Y. Pharmacomechanical thrombectomy in the management of deep vein thrombosis using the cleaner device: an initial single-center experience. Ann Vasc Surg. 2015;29(4):670-4.

4. Fleck D, Albadawi H, Shamoun F, Knuttinen G, Naidu S, Oklu R. Catheter-directed thrombolysis of deep vein thrombosis: literature review and practice considerations. Cardiovasc Diagn Ther. 2017;7(Suppl 3):228-37.

5. Lu Y, Chen L, Chen J, Tang T. Catheter-directed thrombolysis versus standard anticoagulation for acute lower extremity deep vein thrombosis: a meta-analysis of clinical trials. Clin Appl Thromb Hemost. 2018;24(7):1134-43.

6. Crowner JR, Marston W. Percutaneous thrombectomy using a novel single-session device for acute ilio-caval deep vein thrombosis. J Vasc Surg Cases Innov Tech. 2019;5(3):302-4.

7. Dopheide JF, Sebastian T, Engelberger RP, Haine A, Kucher N. Early clinical outcomes of a novel rheolytic directional thrombectomy technique for patients with iliofemoral deep vein thrombosis. VASA. 2018;47(1):56-62.

8. Stain M, Schönauer V, Minar E, Bialonczyk C, Hirschl M, et al. The post-thrombotic syndrome: risk factors and impact on the course of thrombotic disease. J Thromb Haemost. 2005 Dec;3(12):2671-6.

9. ISTH Steering Committee for World Thrombosis Day. Thrombosis: a major contributor to the global disease burden. J Thromb Haemost. 2014;12(10):1580-90.

10. Roumen-Klappe EM, den Heijer M, Janssen MC, van der Vleuten $\mathrm{C}$, Thien $\mathrm{T}$, et al. The post-thrombotic syndrome: incidence and prognostic value of non-invasive venous examinations in a six-year follow-up study. Thromb Haemost. 2005;94(4):825-30.

11. Comerota AJ, Grewal N, Martinez JT, Chen JT, Disalle R, et al. Postthrombotic morbidity correlates with residual thrombus following catheter-directed thrombolysis for iliofemoral deep vein thrombosis. J Vasc Surg. 2012;55(3):768-73.

12. Grewal NK, Martinez JT, Andrews L, Comerota AJ. Quantity of clot lysed after catheter-directed thrombolysis for iliofemoral deep venous thrombosis correlates with postthrombotic morbidity. J Vasc Surg. 2010;51(5):1209-14.

13. Kahn SR, Hirsch A, Shrier I. Effect of postthrombotic syndrome on health-related quality of life after deep venous thrombosis. Arch Intern Med. 2002;162(10):1144-8.

14. Kahn SR, Shbaklo H, Lamping DL, Holcroft CA, Shrier I, et al. Determinants of health-related quality of life during the 2 years following deep vein thrombosis. J Thromb Haemost. 2008;6(7):1105-12
15. Enden T, Haig Y, Kløw NE, Slagsvold CE, Sandvik L, et al. CaVenT Study Group. Long-term outcome after additional catheter-directed thrombolysis versus standard treatment for acute iliofemoral deep vein thrombosis (the CaVenT study): a randomised controlled trial. Lancet. 2012;379(9810):31-8.

16. Vedantham S, Goldhaber SZ, Julian JA, Kahn SR, Jaff MR, et al. ATTRACT trial investigators. Pharmacomechanical Catheter-directed thrombolysis for deep-vein thrombosis. N Engl J Med. 2017;377(23):2240-52.

17. Kearon C, AkL EA, Ornelas J, Blaivas A, Jimenez D, et al. Antithrombotic therapy for VTE disease: CHEST guideline and expert panel report. Chest. 2016;149(2):315-52.

18. Kahn SR, Comerota AJ, Cushman M, Evans NS, Ginsberg JS, et al. American Heart Association Council on Peripheral Vascular Disease, Council on Clinical Cardiology, and Council on Cardiovascular and Stroke Nursing. The postthrombotic syndrome: evidence-based prevention, diagnosis, and treatment strategies: a scientific statement from the American Heart Association. Circulation. 2014;130(18):1636-61.

19. Soosainathan A, Moore HM, Gohel MS, Davies AH. Scoring systems for the post-thrombotic syndrome. J Vasc Surg. 2013;57(1):254-61.

20. Comerota AJ, Kearon C, Gu CS, Julian JA, Goldhaber SZ, et al. ATTRACT Trial Investigators. Endovascular Thrombus Removal for Acute Iliofemoral Deep Vein Thrombosis. Circulation. 2019;139(9):1162-73.

\section{History}

Submitted: 30.09.2020

Accepted after revision: 09.11.2020

Published online: 09.12.2020

\section{Conflicts of interests}

I am a shareholder and medical advisor to VETEX.

\section{ORCID}

Gerard O'Sullivan

(iD) https://orcid.org/0000-0003-3215-4306

\section{Correspondence address}

Prof. Gerard O'Sullivan

Galway University Hospitals

Interventional Radiology

Newcastle Road

Galway H91 YR71

Ireland

gerard.osullivan2@hse.ie 